\title{
MICROSTRUCTURE AND PERMEABILITY OF DRIED PRODUCT FOR FROZEN AQUEOUS SOLUTION
}

\author{
Hideo SHINAGAWA AND YUII KAWAMURA \\ Department of Chemical Engineering, Hiroshima University, Hiroshima 730
}

\section{Introduction}

Freeze-drying has beeen used for the dehydration of heat-sensitive substances, and there have appeared various experimental works ${ }^{11}$ on many kinds of foodstuffs and model materials such as packed beds of glass beads or brick, saturated with water in their void portion.

Most of these studies have developed analyses on the basis of the uniform retreat model of subliming interface. Recently, Toei et al. ${ }^{2)}$ made a valuable study of the non-uniform retreat of subliming interface, and their analytical result agreed well with experimental results for model samples.

However, only a few studies concerning freezedrying of aqueous solutions accompanied by recrystallization of a solute during freezing have been reported, in spite of the fact that freeze-drying is of practical use in the production of medicine and other heatsensitive materials.

As a result the drying characteristics for samples of the above solutions remain unknown. As the first step to elucidate the mechanism of drying, the present work was undertaken experimentally to find the mechanism of freeze-drying, and to examine the effects of freezing conditions and solute concentration on the permeability of the dried layer. $\mathrm{KNO}_{3}$ aqueous solution was chosen as sample because of larger variation of crystal structure resulting from larger variation of solubility of $\mathrm{KNO}_{3}$ in water with temperature.

\section{Experiment}

The drying chamber used was a stainless steel cylinder of $150 \mathrm{~mm}$ inner diameter and $200 \mathrm{~mm}$ height.

The main conduit of the evacuation system consisted of 2 in.-ID stainless steel tube.

Heat necessary for sublimation of ice was supplied by radiation from the heater underlying a sample pan.

The sample pan consisted of a $30 \mathrm{~mm}$-ID (height $11.5 \mathrm{~mm}$ ) acrylic resin cylinder and $34 \mathrm{~mm} \phi$ (thickness $1.5 \mathrm{~mm}$ ) brass plate.

The drying experiments for the frozen samples of

Received November 7, 1978. Correspondence concerning this article should be addressed to H. Shinagawa.
$\mathrm{KNO}_{3}$ aqueous solution were made under the constant heater current $I=0.50 \mathrm{Amp}$.

The weight change of the sample was measured by a displacement transformer. The vapor pressure in the drying chamber was measured by an Alphatron vacuum gauge calibrated with reference to the relationship of the equilibrium vapor pressure-temperature.

The temperatures in the sample were measured with four $40 \mu$-diameter copper-constantan thermocouples arranged in parallel with the base plate of the sample pan.

Drying experiments were carried out for samples of concentration $C_{0}=16.7 \mathrm{wt} \%$ frozen at freezing-bath temperatures of $t_{c}=-75,-49,-32^{\circ} \mathrm{C}$, and for samples of $C_{0}=28.6$ and $37.5 \%$ frozen at $t_{\mathrm{c}}=-75^{\circ} \mathrm{C}$.

Variations of $t_{\mathrm{e}}$ during freezing were within $\pm 2^{\circ} \mathrm{C}$.

\section{Results and Discussion}

\section{1 Crystallographic characteristics of dried layer}

The dried product appeared to be fibrous by nakedeye observation, and was soft like cotton, but not fragile.

Photomicrographs of the dried products are shown in Fig. 1. The photomicrographs of Fig. 1(a), 1(b) show the same sample.

It can be seen from Fig. 1 that distribution of crystal size is significant for the same sample, and that the dried layer consists of fine needle crystals, crystals coagulated in bundles of the needle-like crystals, and long plate-like crystals or crystals with round surface.

It was observed as shown in Fig. 1 that the higher the freezing temperature (or the slower the freezing rate) and the higher the solution concentration $C_{0}$, the greater the size of crystals and the degree of coagulation, rigidity and roundness. The same trend with regard to crystal characteristics was observed slightly along the surface to the bottom of the sample. 2. 2 Dependence of permeability on freezing condition and solution concentration

The relationship between specific resistance $\Delta p / R$ ( $\Delta p=$ pressure drop across dried layer, $R=$ drying rate per unit cross-sectional area) and thickness of dried layer $\delta$ is given as follows if the subliming interface retreats uniformly and heat-mass transfers are in pseudo-steady state. 


$$
\left(p_{s}-p_{v}\right) / R=\Delta p / R=L(1-x) / k=\delta / k
$$

where $k$ is mass transfer coefficient (or permeability) for vapor flow through the dried layer.

Variations of $\Delta p / R$ and $t_{s}$ with $\delta$ obtained from the drying data are shown in Fig. 2.

The interface temperatures were determined by enveloping the inflection points appearing in the relationship of temperature measured by thermocouple vs. drying time.

It can be seen from Fig. 2 that the relationship between $\Delta p / R$ and $\delta$ is linear except in the final stage of drying. This linear relationship shows that vapor transfer through the dried layer lies in the free molecule regime, and that transfer resistance at the interface can be neglected.

The values of $k$ determined from the slopes of these straight lines are given in Table 1. Table 1 contains observed values of permeability by a permeabilitymeasuring apparatus, using samples of complete dryness. It can be seen from Table 1 that both values agree fairly well, and that freezing at higher $t_{c}$ provides samples of higher permeability; $k$ for the samples of $C_{0}=28.6$ and $37.5 \%$ are smaller than for $C_{0}=16.7 \%$ but the degree of decrease in $k$ is not so large as suggested by the decrease of porosity.

The effect of $t_{c}$ on $k$ may result from the decrease of specific surface of the dried porous layer (the resistance to vapor flow through the dried porous layer) accompanied by the formation of larger crystals with the rise of $t_{c}$. The effect of $C_{0}$ on $k$ may also be caused by the decrease of specific surface area accompanied by the formation of more stable and rigid crystals. This decrease compensates a significant decrease of permeability resulting from the decrease of porosity.

\section{Conclusions}

From the present work the following conclusions are drawn.

1) The increase of freezing rate resulted in a significant decrease of permeability. A small decrease of permeability with increase of solution concentration was observed, contrary to the significant decrease of porosity.

2) The effects mentioned in (1) might be caused by the variation of structure of the dried layer with freezing condition and solution concentration.

\section{Nomenclature}

$$
\begin{aligned}
& C_{0}=\text { concentration of sample solution } \\
& I \quad=\text { electric current } \\
& k \quad=\text { permeability of dried porous layer } \\
& \begin{array}{ll}
L & =\text { height of sample } \\
p_{\mathrm{av}} & =\text { arithmetic mean pressure, defined by }
\end{array} \\
& \left(p_{s}+p_{y}\right) / 2
\end{aligned}
$$

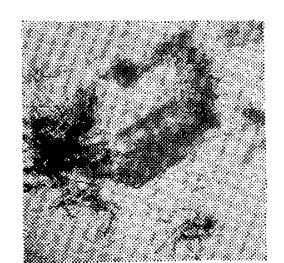

$C_{0}=16.7 \%, t_{c}=-76^{\circ} \mathrm{C}$

(a)

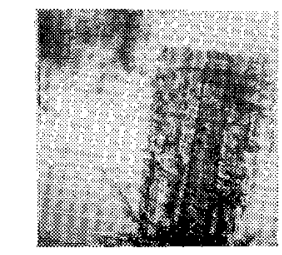

$C_{0}=16.7 \%, t_{c}=-32^{\circ} \mathrm{C}$

(c)

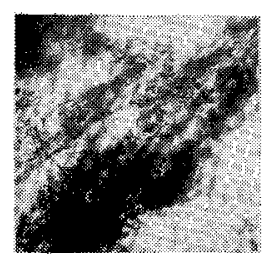

$C_{0}=16.7 \%, t_{c}=-76^{\circ} \mathrm{C}$

(b)

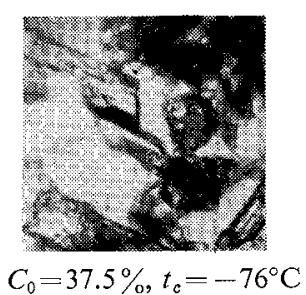

(d) $(\times 15)$

Fig. 1 Photomicrograph of dried layer

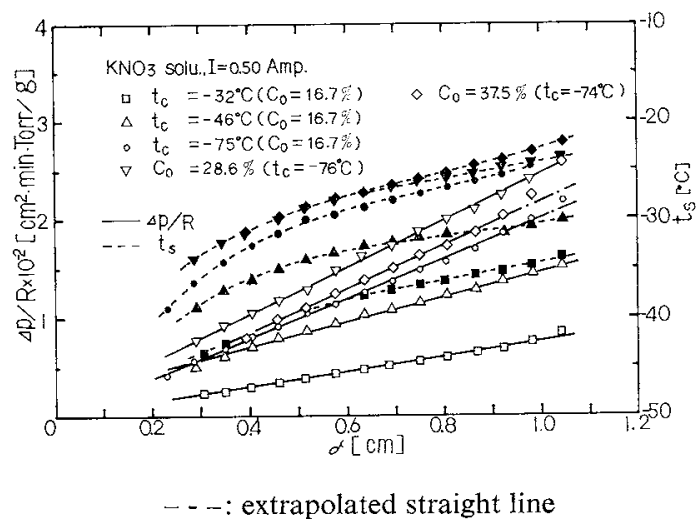

Fig. 2 Variation of $\Delta p / R$ and $t_{s}$ with $t_{c}$ and $C_{0}$

Table 1 Permeability of dried layer for samples of $\mathrm{KNO}_{3}$ aqueous solution

\begin{tabular}{cccc}
$\begin{array}{c}C_{0} \\
{[\mathrm{wt} \%]}\end{array}$ & $\begin{array}{c}t_{c} \\
{\left[{ }^{\circ} \mathrm{C}\right]}\end{array}$ & $\begin{array}{c}\varepsilon \\
{[-]}\end{array}$ & $\begin{array}{c}k \times 10^{3} \\
{[\mathrm{~g} / \mathrm{cm} \cdot \mathrm{min} \cdot \text { Torr }]}\end{array}$ \\
\hline 37.5 & -74 & 0.77 & 4.66 \\
28.6 & -76 & 0.84 & 4.28 \\
16.7 & $-75^{*}$ & $0.92^{*}$ & $5.50^{*}, 5.83 \pm 0.44^{* *}$ \\
16.7 & -49 & 0.91 & 7.43 \\
16.7 & -32 & 0.91 & 13.20 \\
\hline
\end{tabular}

* Averaged value of three different drying experiments, under the same freezing conditions.

* Directly measured value for the sample with complete dryness by permeability measuring equipment, $p_{a}<0.55$ Torr.

$p_{s} \quad=$ vapor pressure at the subliming interface or equilibrium vapor pressure corresponding to $t_{s}$

$p_{v} \quad=$ vapor pressure in the drying chamber

[Torr]

$R \quad=$ drying rate per cross-sectional area of sample $\quad\left[\mathrm{g} / \mathrm{cm}^{2} \cdot \mathrm{min}\right]$

$t_{c}=$ temperature of coolant $\quad\left[{ }^{\circ} \mathrm{C}\right]$

$\begin{array}{lll}t_{s} & =\text { temperature at the subliming interface } & {\left[{ }^{\circ} \mathrm{C}\right]}\end{array}$

$x \quad=$ residual fraction of ice $\quad[-]$ 
2) Toei, R., M. Okazaki and M. Asaeda: J. Chem. Eng. Japan, 8, 282 (1975).

Literature Cited

1) King, C. J.: "Freeze-Drying of Foods", Butterworths

(A part of this work was presented at the Annual Meeting of The Soc. Chem. Engrs., Japan, Hiroshima, 1977.)

\title{
RELATION BETWEEN INTENSITY OF SEGREGATION AND DEGREE OF SEGREGATION IN STIRRED TANK REACTOR
}

\author{
Masaharu TAKAO, Osamu NOMOTO, Yasuhiro MURAKAMI and Yuji SATO \\ Department of Chemical Engineering, Kyushu University, Fukuoka 812
}

After two criteria for the degree of mixing, namely $I s$ and $J$, were defined by Danckwerts ${ }^{3}$ and developed by Zwietering ${ }^{14}$, their physical meanings and relations were studied. Evangelista ${ }^{4)}$ regarded $I s=1 /(1+\beta \tau)$ as $J$, while $\mathrm{Rao}^{97}$ pointed out that $I S$ and $J$ were two different parameters, not to be confused with one another. It was confirmed in the previous paper ${ }^{12)}$ that $I S$ equals $J$ in many models ${ }^{7}$. The aim of the present paper is to provide an expression of $I S$ and $J$ in the partially segregated flow reactor of unsteady state. This allows clarification of their physical meaning and their relation in batch, semibatch and steady-flow stirred-tank reactors under the condition of perfect macro-mixing.

$I s$ for two fluids $A$ and $B$ was defined by Danckwerts $^{3)}$ as

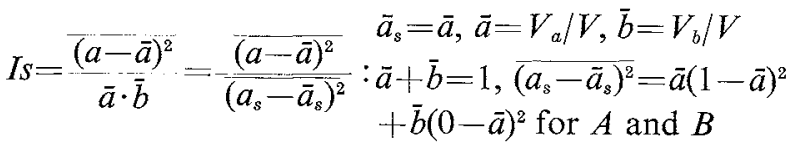

$\overline{\left(a_{s}-\bar{a}_{s}\right)^{2}}$ means the variance of dimensionless concentration $C_{\alpha} / C_{a 0}$ under complete segregation.

$J$ was defined as

$$
J=\frac{\overline{\left(\alpha_{p}-\bar{\alpha}\right)^{2}}}{(\alpha-\bar{\alpha})^{2}}=\frac{\overline{\left(\alpha_{p}-\bar{\alpha}_{p}\right)^{2}}}{\left(\alpha_{p s}-\overline{\left.\alpha_{p s}\right)^{2}}\right.}: \begin{aligned}
& \alpha=\alpha_{p s}, \bar{\alpha}=\bar{\alpha}_{p}=\bar{\alpha}_{p s} \\
& \alpha=\text { age of molecule }
\end{aligned}
$$

Some calculations for $J$ have been proposed for Zwietering's model ${ }^{14)}$ elsewhere ${ }^{10)}$. In numerical calculation $\alpha$ and $\bar{\alpha}$ equal $\alpha_{p s}$ and $\bar{\alpha}_{p s}$, respectively. It is reasonable to transform the variance of age of molecule into that of fluid element at a point under complete segregation.

In the previous paper ${ }^{11}$, it was attempted to grasp

Received January 5, 1979. Correspondence concerning this article should be addressed to M. Takao. systematically many micromixing models ${ }^{2,5}$ by applying the macroscopic population balance ${ }^{6,8)}$. Introduce the mass exchange type model ${ }^{1,5)}$ to the mixing rate term (birth-and-death term, $\vec{B}-\bar{D}$ ) in it. The mass balance of concentration distribution is as follows.

$$
\begin{aligned}
& \frac{1}{V} \cdot \frac{\partial}{\partial t}[V p(c, t)]+\frac{\partial}{\partial c}\left[\frac{d c}{d t} \cdot p(c, t)\right]-\frac{\partial}{\partial c}\left[k_{m}(c-\bar{c}) p(c, t)\right] \\
& =\left[Q_{i} p_{i}(c, t)-Q_{o} p(c, t)\right] / V ; \bar{c}=\bar{c}(t)=\int_{0}^{\infty} c \cdot p(c, t) d c
\end{aligned}
$$

where $d c / d t=-r(c)$ means the rate of change in concentration due to chemical reaction.

The rate of change in volume is

$$
d V / d t=Q_{i}(t)-Q_{o}(t)
$$

Neglect the reaction term of Eq. (3) to multiply by $c \cdot d c$. Integrate over the reactor volume. The first moment of $\bar{c}$ can be derived as

$$
\begin{aligned}
\frac{d(V \bar{c})}{d t}= & \left(Q_{i} \bar{c}_{i}-Q_{0} \bar{c}\right)+V\left[c k_{m}(c-\bar{c}) p(c, t)\right]_{c=0}^{c=\infty} \\
& -V k_{m} \int_{0}^{\infty}(c-\bar{c}) p(c, t) d c: \bar{c}_{i}=\int_{0}^{\infty} c p_{i}(c, t) d c
\end{aligned}
$$

The 2 nd term of the right-hand side in Eq. (5) vanishes, because it becomes zero at $c=0$ and $c=\infty$, namely $p(\infty, t)=0$. The third term is also zero. Equation (5) is simplified as

$$
d(V \bar{c}) / d t=Q_{i} \bar{c}_{i}-Q_{o} \bar{c} \text { or } d \bar{c} / d t=Q_{i}\left(\bar{c}_{i}-\bar{c}\right) / V
$$

The relation of 2 nd moment is derived as

$$
\frac{d\left(\overline{V c^{2}}\right)}{d t}=Q_{i} \overline{c_{i}^{2}}-Q_{0} \overline{c^{2}}-2 k_{m} V \int_{0}^{\infty} c(c-\bar{c}) p(c, t) d c
$$

Utilize Eq. (6), and subsitute $\operatorname{Varc}=\overline{c^{2}}-(\bar{c})^{2}$ and $\operatorname{Varc}_{i}=\overline{c_{i}{ }^{2}}-2 \overline{c_{i}} \bar{c}+(\bar{c})^{2}$. Equation (7) is arranged as 